2008-09-01

\title{
Ultracompact AWG Using Air-Trench Bends With Perfluorocyclobutyl Polymer Waveguides
}

\author{
Seunghyun Kim \\ Gregory P. Nordin \\ nordin@byu.edu \\ John Ballato \\ Yongbin Lin \\ Nazli Rahmanian
}

See next page for additional authors

Follow this and additional works at: https://scholarsarchive.byu.edu/facpub

Part of the Electrical and Computer Engineering Commons

\section{Original Publication Citation}

Y. Lin, S. Kim, N. Rahmanian, G. P. Nordin, C. Topping, D. W. Smith, Jr., and J. Ballato, "Ultracompact AWG using air-trench bends with perfluorocyclobutyl polymer waveguides," IEEE J. Lightwave Techn. 26(17), pp. 362-37 (28).

\section{BYU ScholarsArchive Citation}

Kim, Seunghyun; Nordin, Gregory P.; Ballato, John; Lin, Yongbin; Rahmanian, Nazli; Smith, Dennis W.; and Topping, Chris, "Ultracompact AWG Using Air-Trench Bends With Perfluorocyclobutyl Polymer Waveguides" (2008). Faculty Publications. 892.

https://scholarsarchive.byu.edu/facpub/892 


\section{Authors}

Seunghyun Kim, Gregory P. Nordin, John Ballato, Yongbin Lin, Nazli Rahmanian, Dennis W. Smith, and Chris Topping 


\title{
Ultracompact AWG Using Air-Trench Bends With Perfluorocyclobutyl Polymer Waveguides
}

\author{
Yongbin Lin, Nazli Rahmanian, Seunghyun Kim, Gregory P. Nordin, Member, IEEE, Chris Topping, \\ Dennis W. Smith, Jr., and John Ballato
}

\begin{abstract}
Using air-trench bends, an ultracompact $8 \times 8$ arrayed waveguide grating (AWG) demultiplexer (200-GHz channel spacing) for wavelength-division multiplexing (WDM) has been designed and fabricated with perfluorocyclobutyl (PFCB) core and clad co-polymers on a polyimide substrate. Compared to a conventional AWG in the same material system, the air-trench bend AWG shrinks the required chip area by a factor of 20 . The decreased size is a factor in reducing the measured thermal shift to $-0.012 \mathrm{~nm} /{ }^{\circ} \mathrm{C}$ and decreasing the polarization-dependent wavelength shift to $1.3 \mathrm{~nm}$.
\end{abstract}

Index Terms-Optical filters, optical planar waveguide components, waveguide arrays, waveguide bends.

\section{INTRODUCTION}

A RRAYED-WAVEGUIDE gratings (AWGs) [1] are key enabling components for high-capacity fiber communication systems based on wavelength-division multiplexing (WDM). Conventional AWGs implemented in low index and low index contrast (LILIC) waveguide material systems (core-clad refractive index contrast $\Delta \mathrm{n} / \mathrm{n} \leq 1.5 \%$ ) rely on curved waveguide bends to achieve waveguide path length differences in the arrayed waveguides. The minimum radius of curvature for LILIC waveguides is at least several millimeters to achieve high optical throughput [2], which fundamentally limits how small such AWGs can be made. The relatively large size of conventional AWG devices not only increases the device manufacturing cost, but also affects AWG device performance. For example, the amount of phase error caused by refractive index fluctuations in the waveguides is directly related to the

\footnotetext{
Manuscript received August 20, 2007; revised January 25, 2008. Current version published December 19, 2008. This work was supported in part by DARPA Grant N66001-04-8933.

Y. Lin and N. Rahmanian are with the Nano and Micro Device Center (NMDC), University of Alabama in Huntsville, Huntsville, AL 35899 USA (e-mail: liny@eng.uah.edu).

S. Kim and G. P. Nordin are with the Electrical and Computer Engineering Department, Brigham Young University, Provo, UT 84602 USA (e-mail: shkim@ee.byu.edu; nordin@ee.byu.edu).

C. Topping is with the Tetramer Technologies, Pendleton, SC 29670 USA (e-mail: ctoppin@clemson.edu).

D. W. Smith, Jr., is with the Department of Chemistry and the Center for Optical Materials Science and Engineering Technologies (COMSET), Clemson University, Clemson, SC 29634 USA (e-mail: dwsmith@clemson.edu).

J. Ballato is with the School of Materials Science and Engineering and the Center for Optical Materials Science and Engineering Technologies (COMSET), Clemson University, Clemson, SC 29634 USA (e-mail: jballat@clemson.edu).

Color versions of one or more of the figures in this paper are available online at http://ieeexplore.ieee.org.

Digital Object Identifier 10.1109/JLT.2008.920091
}

length of the waveguides. Likewise, the amount of thermal wavelength shift and polarization-dependent shift also increase with larger optical path lengths. Hence, dramatic reduction in AWG size is not only beneficial from an economic point of view, but can also yield improvements in performance.

There are basically two approaches that have been reported to reduce AWG size in low refractive index material systems such as silica and various polymers. One is to increase the refractive index contrast from $0.75 \%$ to $1.5 \%$ or even higher. This leads to limited device dimension reduction while posing the challenge of higher loss for fiber-to-chip coupling. An example of this approach is a compact AWG based on silica-on-silicon (SOS) reported by Mizuno et al. [3]. In their work, a 16-channel AWG was fabricated with SOS waveguides having a refractive index contrast of $1.5 \%$ and with integrated spot-size converters to reduce the coupling loss from fiber to chip. The reported device measured $16 \times 16 \mathrm{~mm}^{2}$. The limitation of this approach is that it is based on curved waveguide bends, which make it difficult to further reduce device size because of limitations on how much the refractive index contrast can be increased. A second approach is to use abrupt waveguide bends such as corner mirrors or air-trench bends to reduce or eliminate the need for large radius bends. An example is the ultrasmall arrowhead silica AWG with V-shaped bends in the arrayed waveguides reported by Suzuki and Tsuda [4]. V-shaped bends are realized with an elliptic silver mirror along with curved waveguide bends. By using the v-shape waveguide bend, the authors greatly reduce the length of the curved waveguide bends in the arrayed waveguide region, thus dramatically reducing device dimensions. However, the input and output waveguide sections still rely on curved waveguide bends which limits the possible overall size reduction.

In this paper, we demonstrate an ultracompact AWG that totally avoids curved waveguide bends by using $45^{\circ}$ air-trench bends in both the arrayed waveguide and input/output waveguide regions. We implement an $8 \times 8$ AWG in a perfluorcyclobutyl (PFCB) polymer (Tetramer Technologies, Pendleton, SC) waveguide material system [5]-[7] with a $1.3 \%$ refractive index contrast on polyimide substrates. The chip area for our air-trench bend PFCB AWG is $8.4 \mathrm{~mm} \times 1.5 \mathrm{~mm}$, which is only $1 / 20$ the size of a comparable AWG using curved waveguides in the same material system. To the best of our knowledge, this is the smallest $8 \times 8$ AWG device that has been reported for LILIC material systems.

While the results reported in this paper are demonstrated in a PFCB waveguide material system, similar results should be obtainable in other LILIC materials such as silica. PFCB was 


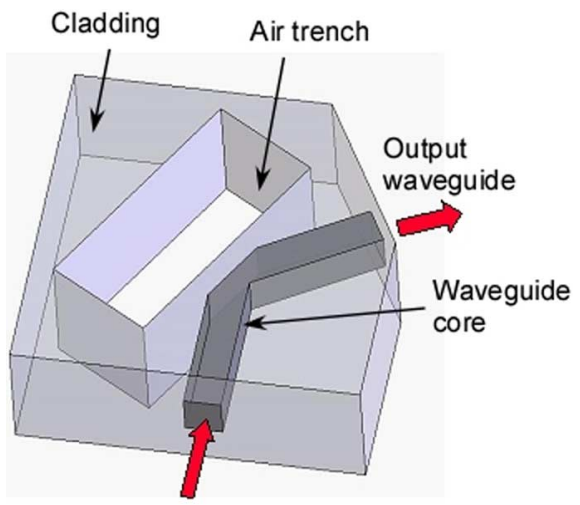

Input waveguide

Fig. 1. Three-dimensional schematic illustration of an air-trench $45^{\circ}$ bend [17], [18].

chosen because of its unique combination of low loss in the near infrared and its processability, and because it has been shown to offer a wide range of optical functionalities including doping with rare earth [8], inorganic nanoparticle [9]-[11], organic chromophore [12], and quantum dot light emitters [13], nonlinear chromophores [14], and liquid crystalline moieties [15]. This diversity of optical functionalities in one host is useful for the development of other active and passive devices. Nonetheless, the success of our approach to reduce AWG size through use of air trench bends depends only on the ability to etch smooth, highly vertical interfaces that are precisely positioned with respect to each waveguide bend intersection. A key advantage of using air-trench bends is that the waveguide bend structure is independent of both the refractive index and the refractive index contrast between the core and clad materials since it is based on total internal reflection (TIR). Hence, it may be used even for very low refractive index contrast waveguides (such as $0.5 \%$ or $0.75 \%$ ) in silica or other polymers as long as the requisite TIR interfaces can be etched and properly positioned.

\section{WAVEGUide AIR-TRENCH BENDS AND AWG}

A schematic diagram of an air-trench $45^{\circ}$ waveguide bend is shown in Fig. 1. It consists of input and output single-mode waveguides embedded in a cladding, and an air-trench that has a vertical sidewall positioned near the intersection of the center lines of the input and output waveguides. For our PFCB waveguides the cross-sectional dimension of the core is $3.6 \mu \mathrm{m} \times 3.6 \mu \mathrm{m}$ to ensure single-mode propagation at a wavelength of $1550 \mathrm{~nm}$. The core refractive index is 1.4836 for TE polarization (electric field in the plane of the substrate) and 1.4816 for TM polarization (electric field out of the plane of the substrate). The cladding refractive indexes for TE and TM polarization are 1.4644 and 1.4625 , respectively. The core and clad are comprised of the same PFCB copolymers but in different proportions.

We have done extensive design, analysis and characterization of air-trench bends for low refractive index waveguide material systems [16] and have demonstrated high efficiency $45^{\circ}$ air-trench bends in PFCB waveguides on silicon substrates [17], [18]. The measured bend optical efficiency for TE and TM po-

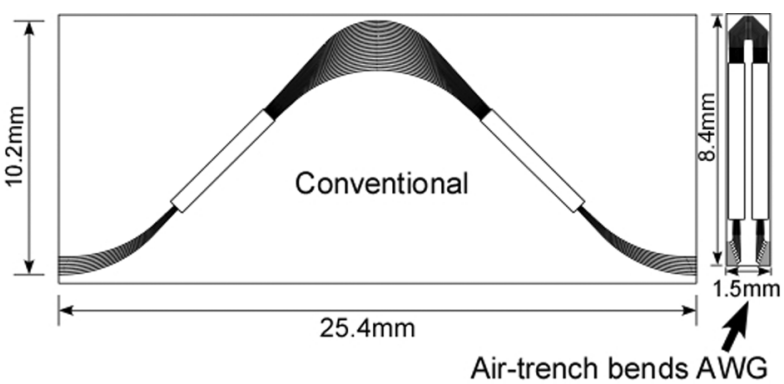

Fig. 2. Layouts for a conventional AWG and an air-trench bend AWG for PFCB waveguides with a refractive index contrast of $1.3 \%$.

larization is $97.2 \%$ and $96.2 \%$ [bend loss of 0.124 (TE) and 0.166 (TM) dB/bend] [18], respectively. This compares favorably with three-dimentional finite-difference time-domain (3DFDTD) simulation predictions of $98.5 \%$ (TE) and $98.0 \%$ (TM) when the Goos-Hanchen shift is properly accounted for [18]. The key factors in obtaining such high measured efficiencies are 1) properly designed bends (the bend angle must be selected to insure that all of the angular spectrum components of the waveguide mode undergo TIR as discussed in [16]), 2) vertical and smooth etched sidewalls, and 3) very accurate positioning (typically within $100 \mathrm{~nm}$ ) of the etched interface. As discussed in [17] and [18], we have developed fabrication processes to achieve these criteria for PFCB waveguides based on inductively coupled plasma reactive ion etching (ICP RIE) and electron beam lithography (EBL) in a scanning electron microscope (SEM).

A schematic layout of our ultracompact AWG using air-trench bends is shown in Fig. 2 at the same scale with one for a conventional AWG designed for the same materials and design parameters. As illustrated in Fig. 3, the footprint of our ultracompact AWG is minimized by applying air-trench bends to the input and output waveguides as well as the arrayed waveguide region, thus totally avoiding large radius curved waveguide bends. The size of the air-trench bend AWG is $8.4 \mathrm{~mm} \times 1.5 \mathrm{~mm}$, which is $1 / 20$ that of the conventional design.

Our $8 \times 8$ air-trench bend AWG is designed to have a channel spacing of $200 \mathrm{GHz}(1.6 \mathrm{~nm})$. The path length difference between adjacent waveguides in the arrayed waveguide region is $110 \lambda$, which results in a free spectral range (FSR) of $1741.33 \mathrm{GHz}(14 \mathrm{~nm})$. The optical path length difference between neighboring waveguides $(\Delta L)$ is achieved by carefully adjusting the spacing of the waveguides and the positioning of the bends. The size of the air trenches in this experiment is $50 \mu \mathrm{m} \times 8 \mu \mathrm{m}$. Due to the size of the air trenches and the waveguide mode profile, the minimum waveguide spacing is set to $35 \mu \mathrm{m}$ in order to avoid clipping the waveguide mode with the corner of an adjacent air trench. The parameters of the air-trench bend AWG are given in Table I. The input and output waveguides are indexed from top to bottom with waveguides 1 and 8 labeled in Fig. 3.

\section{FABRICATION}

We have fabricated air-trench bend AWGs in PFCB on polyimide substrates (OptiCOMP Networks, Cambridge, MA). The 


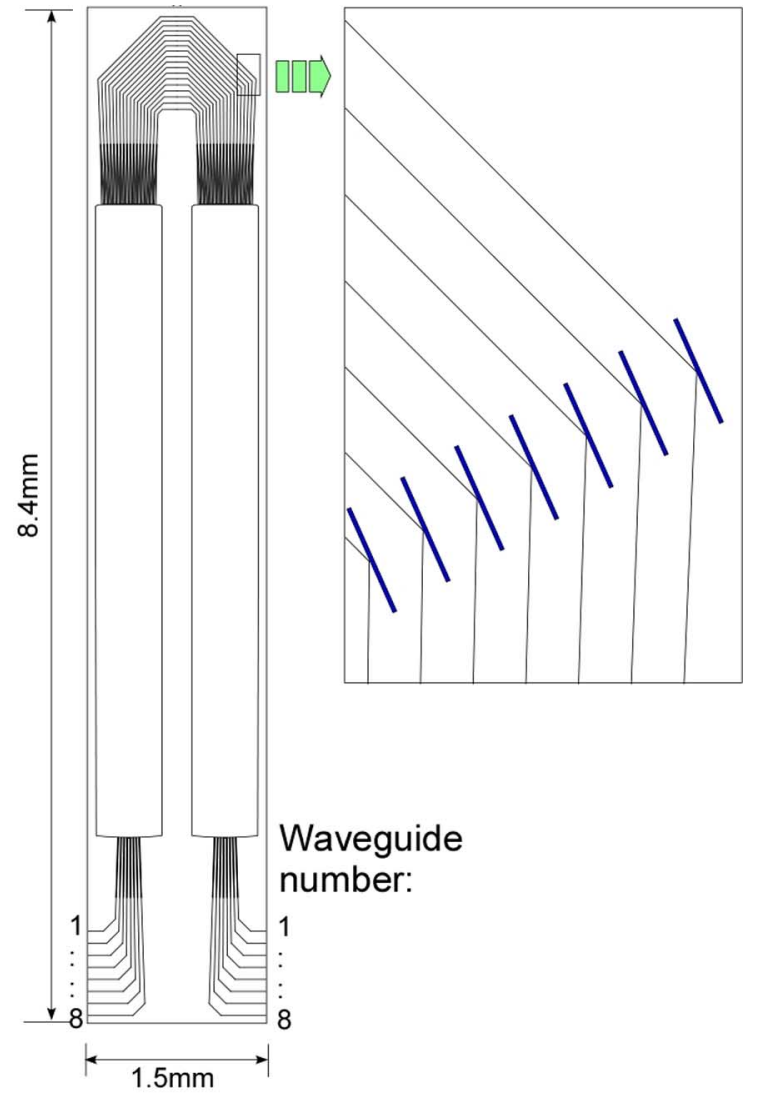

Fig. 3. Air-trench bend AWG. Light enters an input waveguide on the bottom left of the figure and exits through the output waveguides on the bottom right of the figure. The zoomed-in region shows the air trench locations for some of the arrayed waveguides.

TABLE I

DESIGN PARAMETERS FOR AIR-TRENCH BEND AWG

\begin{tabular}{|l|l|l|}
\hline Symbol & Parameter & Value \\
\hline $\mathrm{L}_{\mathrm{f}}$ & Focal length & $5213.992 \mu \mathrm{m}$ \\
\hline$\lambda_{\mathrm{o}}$ & Center wavelength & $1550 \mathrm{~nm}$ \\
\hline $\mathrm{n}_{\mathrm{c}}$ & Array channel index & 1.4836 \\
\hline $\mathrm{n}_{\mathrm{s}}$ & Slab refractive index & 1.4836 \\
\hline $\mathrm{n}_{\mathrm{g}}$ & Group index for $\mathrm{n}_{\mathrm{c}}$ & 1.4991 \\
\hline $\mathrm{x}$ & $\begin{array}{l}\text { Output waveguide } \\
\text { spacing }\end{array}$ & $25 \mu \mathrm{m}$ \\
\hline $\mathrm{d}$ & $\begin{array}{l}\text { Grating waveguide } \\
\text { spacing }\end{array}$ & $25 \mu \mathrm{m}$ \\
\hline$\Delta \mathrm{L}$ & $\begin{array}{l}\text { Path-length } \\
\text { difference }\end{array}$ & $114.923 \mu \mathrm{m}$ \\
\hline $\mathrm{m}$ & Diffraction order & 110 \\
\hline$\Delta \lambda$ & Wavelength spacing & $1.6 \mathrm{~nm}$ \\
\hline$\Delta \mathrm{v}$ & Frequency spacing & $200 \mathrm{GHz}$ \\
\hline $\mathrm{FSR}$ & Free spectral range & $1741.33 \mathrm{GHz}$ \\
\hline $\mathrm{N}$ out & Total output channel & $8 \mathrm{Channels}$ \\
\hline $\mathrm{N}$ array & $\begin{array}{l}\text { Total phase-delay } \\
\text { waveguides }\end{array}$ & 18 waveguides \\
\hline
\end{tabular}

fabrication process is adapted from the one reported in [18] for PFCB waveguide air-trench bends on silicon wafers. The reason we choose polyimide instead of silicon as the substrate is that the PFCB on top of silicon often develops cracks in the PFCB film stack during fabrication steps that take place on top of the full
TABLE II

PhysicAl Properties of PFCB, Silicon AND POLYIMIDE

\begin{tabular}{|c|c|c|}
\hline & $\begin{array}{c}\mathrm{CTE} \\
(\mathrm{ppm} / \mathrm{C})\end{array}$ & $\begin{array}{c}\text { Thermal } \\
\text { conductivity } \\
(\mathrm{W} / \mathrm{m} * \mathrm{~K})\end{array}$ \\
\hline PFCB & 60 & \\
\hline Silicon substrate & $2.4-4.2$ & $\sim 150$ \\
\hline Polyimide substrate & 56 & $\sim 0.2$ \\
\hline
\end{tabular}

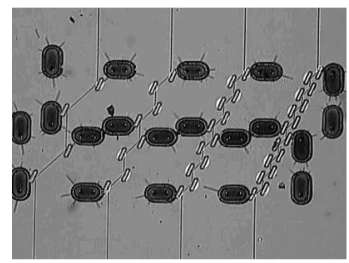

(a)

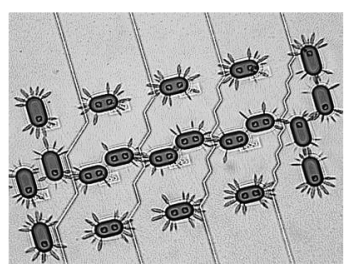

(b)

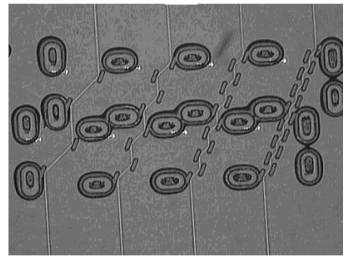

(c)

Fig. 4. (a) Microscope image showing typical cracking for PFCB film stack on silicon. (b) Worst case cracking problem which can occur during spin coating ZEP 520 A. (c) Comparable result for PFCB on polyimide wafers in which cracking is totally avoided.

film stack. Film cracking is normally not a problem when processing the core layer to form single mode waveguides and multimode interference (MMI) slab regions. However, as discussed in [19], fabrication processes that occur on top of the complete film stack (i.e., after the overclad has been applied on top of the waveguides that have been etched in the core layer) often end up promoting the development of cracks owing to stress in the film stack. As pointed out in [19], this stress forms during thermal curing of the PFCB layers because of the coefficient of thermal expansion mismatch between PFCB and silicon. As shown in Table II, the polyimide substrate has a coefficient of thermal expansion (CTE) close to that of PFCB, which greatly diminishes the formation film stress during the curing process.

To illustrate this point, consider the microscope images in Fig. 4. As discussed in [18], the fabrication process for EBLbased air-trench bends has many fabrication steps since the EBL is done in an SEM, which requires separate EBL alignment marks for each write field. We have found that a PFCB film stack on a silicon substrate is likely to exhibit cracking during 


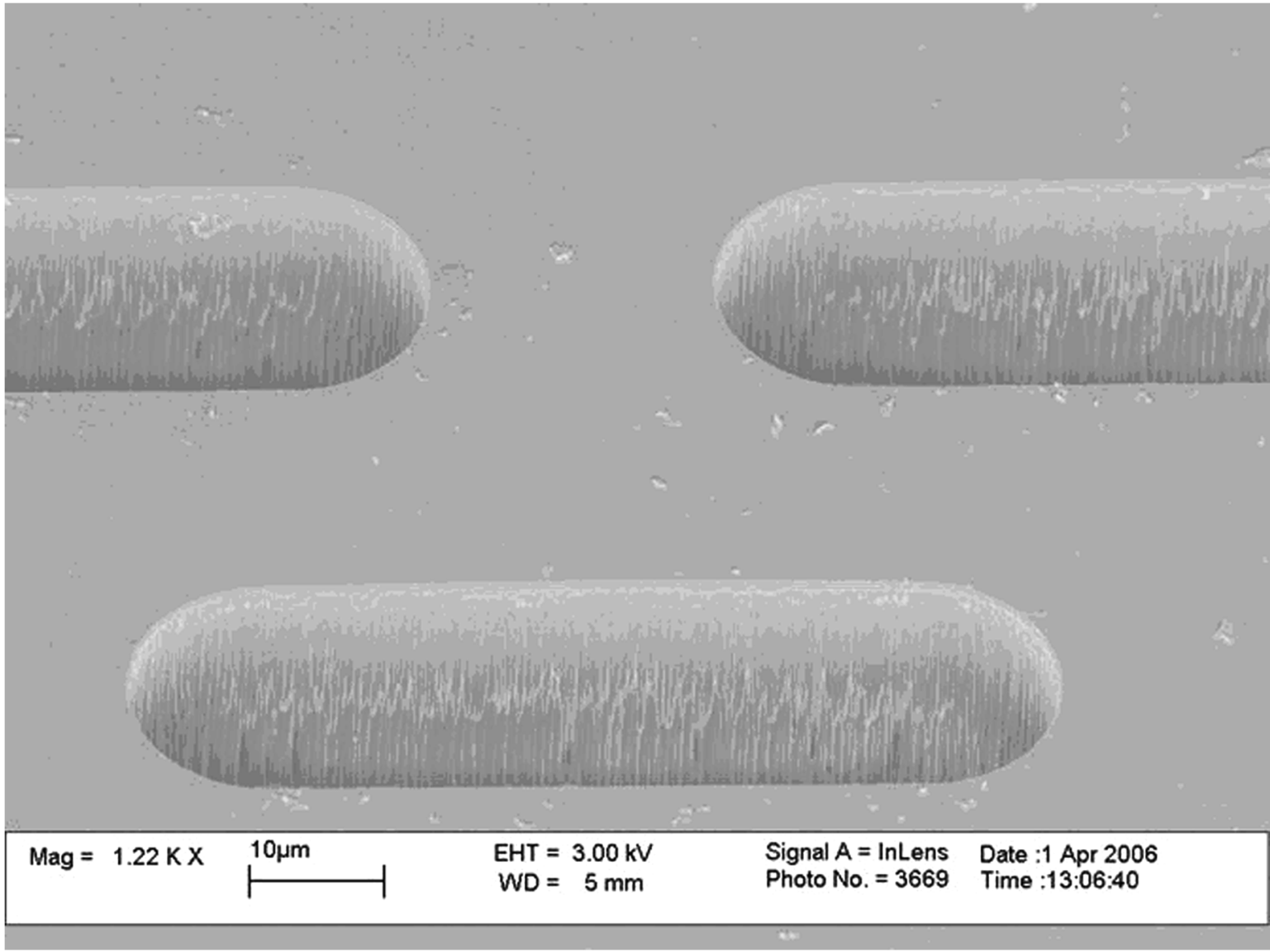

Fig. 5. Etch result for air trenches in PFCB on a polyimide substrate using the step by step etch process discussed in the text.

wet chemical processes such as metal lift-off and electron beam resist spin coating and developing. For example, Fig. 4(a) shows a microscope image of a set of PFCB waveguides on a silicon substrate in which each waveguide has a different number of bends. The dark oval regions are where the EBL alignment marks are located. Typical stress-induced cracking is visible around the oval regions. Fig. 4(b) shows worst case cracking after spin coating an electron beam resist (ZEP 520A). As shown in Fig. 4(c) for the same fabrication process on top of a polyimide wafer, no cracking is present. By using polyimide substrates, the repeatability and yield of the fabrication process is dramatically improved from about $10 \%$ to higher than $80 \%$ in our university cleanroom facilities.

However, use of polyimide substrates has a serious drawback compared to silicon. As indicated in Table II, polyimide has a much lower thermal conductivity than silicon. This results in slower heat removal from the PFCB film during anisotropic ICP RIE etching to form the air trenches, thus raising the PFCB temperature. This in turn increases the rate of the random (isotropic) component of the etch and results in more etch undercut, sidewall roughness, and sidewall tilt. To help reduce PFCB heating during the anisotropic plasma etch process, we used an heuristic approach to develop a step-by-step etch recipe in which the 21 -min etch is divided into 21 one-minute etch steps, separated by $1.5 \mathrm{~min}$ of gas flow with no plasma in the chamber to permit cooling of the sample. A typical etch result for PFCB air trenches on polyimide substrates using this step-by-step etch process is shown in Fig. 5. There is more sidewall roughness compared to PFCB air trenches on silicon substrates (see, for example, [18, Fig. 9]). The effect on optical bend loss is shown in Fig. 6 in which the measured bend loss is $0.31 \pm 0.02 \mathrm{~dB} /$ bend ( $93 \%$ bend optical efficiency) for TE polarization, which is about a factor of two higher than the measured loss for bends on silicon $(0.124 \mathrm{~dB})$. Nonetheless, the only effect of this larger bend loss is an increased device insertion loss. As shown in Fig. 7, the spectral response for PFCB air-trench bends on polyimide still has excellent uniformity across a 100-nm wavelength range (1480 to $1580 \mathrm{~nm}$ ).

We use the step-by-step etch process to fabricate the air trench bends in our AWG devices. Fig. 8(a) shows fabricated arrayed waveguide gratings after the deep air trench etch, and Fig. 8(b) shows the area of fabricated input/output waveguides near the slab waveguide region. Note in Fig. 8(a) that the left- and right-most sets of bends in the arrayed waveguide section have bending angles slightly different from $45^{\circ}$ since the waveguides from the MMI slab regions emerge at slightly different angles. The bend angles vary from $42.66^{\circ}$ to $47.34^{\circ}$. Fig. 9 shows an SEM image of etched air trenches in the arrayed waveguide section. Note that the size of the trenches has been reduced from $70 \mu \mathrm{m} \times 20 \mu \mathrm{m}$ as in Fig. 5 to $50 \mu \mathrm{m} \times 10 \mu \mathrm{m}$ to facilitate closer packing of the waveguides. The sidewall roughness, however, is similar.

Note in Figs. 3 and 8(a) that there are only 18 arrayed waveguides connecting the slab waveguide regions, which is 


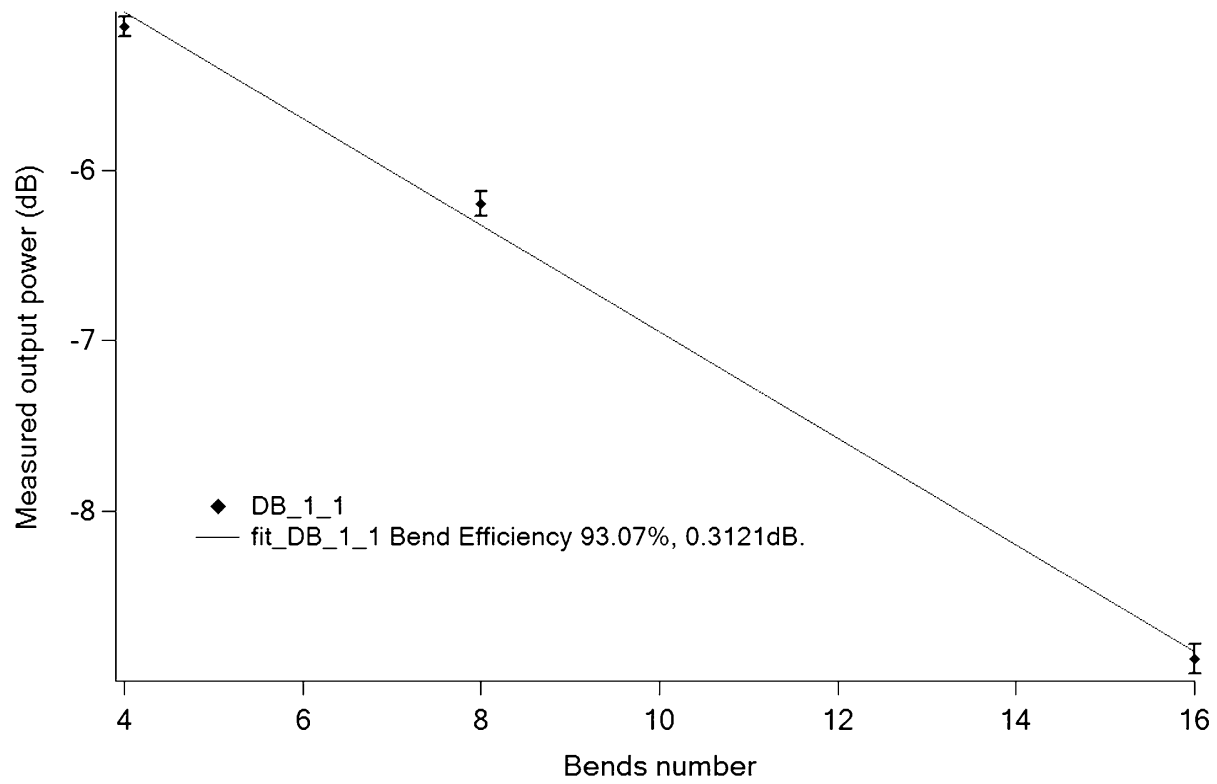

Fig. 6. Measured optical power through a set of equal-length PFCB waveguides on polyimide that have a different number of bends in each waveguide. Details on the measurement method can be found in [9] and [10].

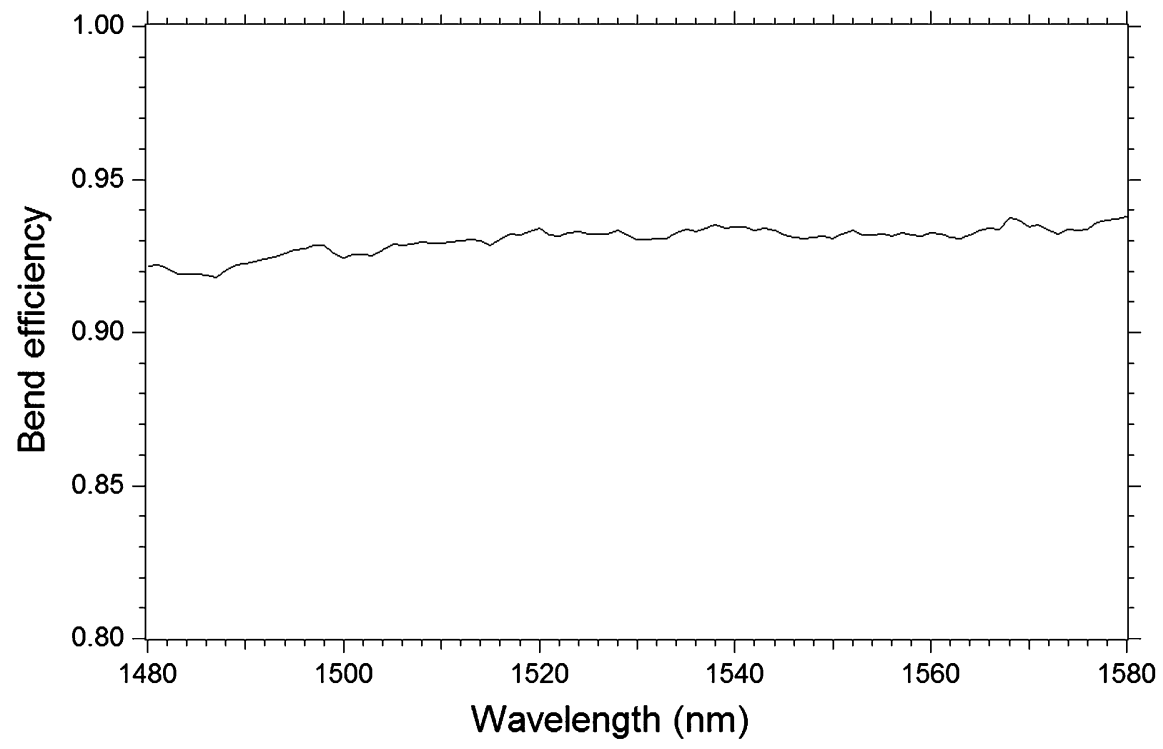

Fig. 7. Spectral response of PFCB waveguide air-trench $45^{\circ}$ bends shows excellent uniformity across a 100-nm wavelength range.

less than the number of arrayed waveguides in a typical $8 \times 8$ AWG design (usually $>50$ ). The small number of arrayed waveguides affects device performance such as insertion loss and channel cross talk. The number of arrayed waveguides in fabricated AWGs is limited by our particular EBL-based fabrication process in which a maximum alignment field size of $1000 \mu \mathrm{m} \times 1000 \mu \mathrm{m}$ is used. Because of the corner area required for the alignment marks and other limitations of our fabrication process, the actual useful field is approximately $760 \mu \mathrm{m} \times 760 \mu \mathrm{m}$. Only 18 waveguides can be placed in this area if a minimum waveguide spacing of $35 \mu \mathrm{m}$ is maintained. This limitation can be easily removed if the air trench bend patterning step is done in a modern optical stepper, which has an alignment accuracy consistent with what can be achieved in our low-end SEM-based EBL.

\section{MEASUREMENT AND Discussion}

Characterization of air-trench bend AWG performance is done in a Newport PM500 Autoalign System with an Agilent 8164A tunable laser (1480-1580-nm tuning range) as the optical source. Light from the laser is coupled into a polarization maintaining (PM) fiber that is butt coupled to one of the AWG input waveguides. The end of the fiber nearest to the sample is rotated to control the polarization state of the light coupled into a waveguide. Light from one of the eight output waveguides is butt coupled to a single mode fiber which is connected to an optical power meter. Two computer-controlled precision 3 axis motion stage stacks with 50-nm accuracy are used to optimize the input and output fiber positions to maximize the power through each waveguide. 


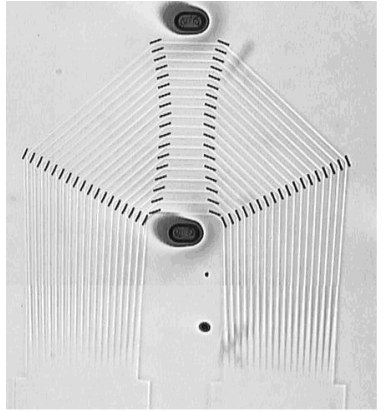

(a)

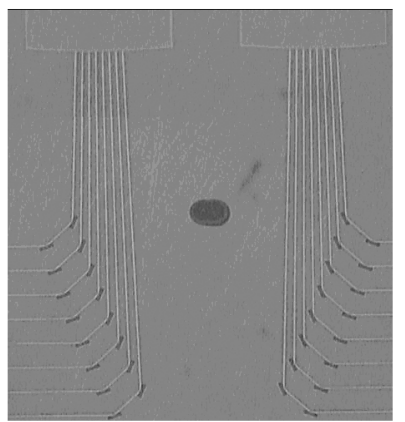

(b)

Fig. 8. AWG microscope images. (a) Fabricated arrayed waveguide gratings after the deep air-trench etch (i.e., the waveguides above the two MMI slab waveguide regions in Fig. 3 and which connect the two MMIs). (b) Input waveguides (left) for the left MMI slab region and output waveguides (right) for the right MMI slab region. Note: the dark oval regions are where the EBL alignment marks are located.

A typical transmission spectrum for a fabricated air-trench bend AWG is shown in Fig. 10 for TE polarization. The input waveguide used in this measurement is one of the center waveguides (waveguide 4). The measured channel spacing is $1.6 \mathrm{~nm}$, and the channel width is $0.65 \mathrm{~nm}$ at $-3 \mathrm{~dB}$.

\section{A. Insertion Loss}

The insertion loss, including on-chip loss and coupling loss between fibers and waveguides, is in the range of 16.2-19.3 dB for all eight output waveguides. This loss can be attributed to several sources. First, the total fiber-to-chip coupling loss is typically $3.2-3.6 \mathrm{~dB}$ in our measurement setup without using refractive index-matching fluid. Second, we estimate that the waveguide propagation loss is $\sim 0.7 \mathrm{~dB}$ based on the typical $0.4-\mathrm{dB} / \mathrm{cm}$ waveguide propagation loss measured in our labs for straight waveguides and the roughly 1.6-cm-long on-chip propagation length (including the MMI slab regions). Third, each waveguide bend introduces some loss. There are eight air-trench bends in each optical path. Based on an average of $0.35-\mathrm{dB} / \mathrm{bend}$ loss, the loss from the air-trench bends for each optical output is $\sim 2.8 \mathrm{~dB}$.

Finally, there is additional loss due to the MMI regions. In order to measure the loss for the MMI slab waveguides, individual MMIs with eight input waveguides and 18 output waveguides are fabricated. A single PM fiber with TE polarized light is butt-coupled to a center input waveguide (waveguide 4), and the light emitted from all 18 waveguides is measured. The total power from these waveguides is $20 \%$ of the input power, which represents a 7- $\mathrm{dB}$ loss. Subtracting $3.2 \mathrm{~dB}$ to account for the total fiber to waveguide coupling losses, we estimate the excess loss for the MMI slab waveguides to be $3.8 \mathrm{~dB}$. As is well known, use of more arrayed waveguides can significantly decrease this loss.

The total loss from the above contributions is $14.5 \mathrm{~dB}$. This leaves 1.7 to $4.8 \mathrm{~dB}$ unaccounted for. We believe that this is due to fabrication defects in the waveguides of the AWG sample used for the measurements presented in this paper.

\section{B. Channel Crosstalk}

The adjacent channel crosstalk is $\leq-7 \mathrm{~dB}$. The channel cross talk is relatively high and is primarily due to two factors. One is the asymmetric sidelobes in the AWG spectrum and the other is the fact that there are only 18 waveguides in the arrayed waveguide section. Fig. 11 shows a typical spectral response for one of the output waveguides. The asymmetric sidelobes in the spectrum is likely caused by a nonlinear phase error related to the Goos-Hanchen shift for the different bend angles in the arrayed waveguide section. As mentioned in Section II, some of the bends in the arrayed waveguide section have bend angles slightly different from $45^{\circ}$. In our initial design and fabrication of AWG samples, we offset the position of all of the bends by the same amount to account for Goos-Hanchen shift. However, this shift should be slightly different depending on the bend angle. We believe that taking this into account in fabricated samples will lead to reduced sidelobe asymmetry and hence lower channel crosstalk.

\section{Thermal Stability}

The spectral response at different temperatures is measured in order to evaluate the thermal stability of air-trench bend AWGs. Fig. 12 shows the measured transmission spectrum of a center output channel (channel 4) at room temperature $\left(21.5^{\circ} \mathrm{C}\right)$ and at a higher temperature $\left(63.2^{\circ} \mathrm{C}\right)$. Over this temperature range the temperature-dependent wavelength shift is $-0.012 \mathrm{~nm} /{ }^{\circ} \mathrm{C}$. This value is much smaller than the thermal shift of the spectral response of conventional AWG devices made with PFCB waveguides on a silicon substrate which is typically $-0.25 \mathrm{~nm} /{ }^{\circ}$ C [20], and is comparable to that of silica based conventional AWGs $\left(-0.012 \mathrm{~nm} /{ }^{\circ} \mathrm{C}\right)$ [21]. This is primarily due to two reasons. As discussed in [22], the first is that use of a substrate with a higher CTE such as polyimide can reduce the thermal wavelength shift of the AWG. The thermal wavelength shift for a conventional AWG with a substrate CTE $\alpha_{\text {sub }}$ can be predicted by [22]

$$
d \lambda_{0} / d T=\left(d n_{c} / d T+n_{c} \alpha_{\mathrm{sub}}\right) \times\left(\lambda_{0} / n_{c}\right)
$$

where $\lambda_{0}$ represents the central wavelength, and $n_{c}$ is the effective refractive index of the waveguide. Based on the PFCB copolymers used in this experiment with $d n_{c} / d T=-1.4 \times$ $10^{-4} / K$ and $\alpha_{\text {sub }}$ for polyimide as given in Table II, the calculated thermal wavelength shift is $-0.059 \mathrm{~nm} /{ }^{\circ} \mathrm{C}$, which is significantly larger than the measured shift for our air trench bend AWG. This leads to the second reason for the small shift, which is the shorter path length in the arrayed waveguides of the 


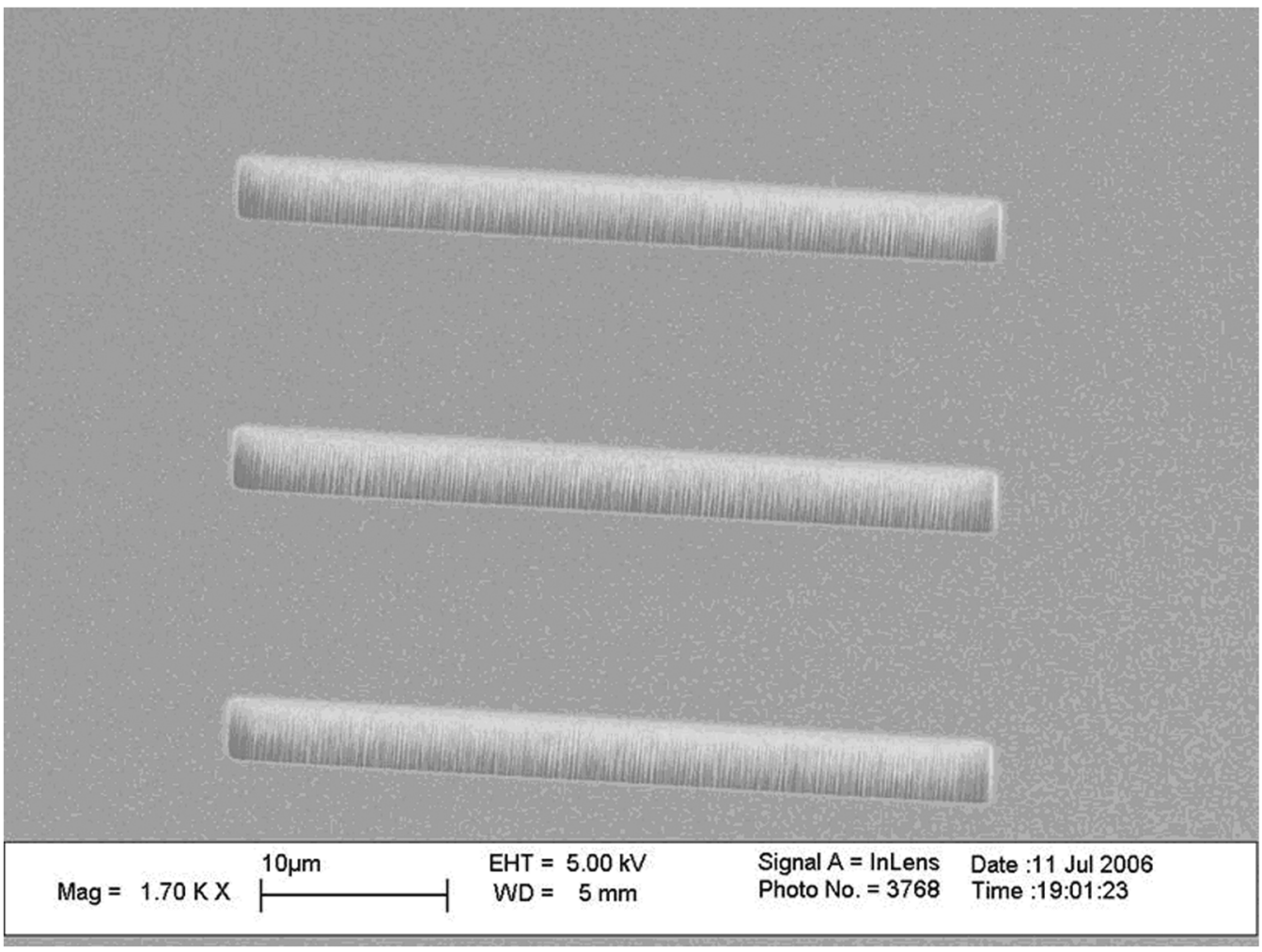

Fig. 9. Etched PFCB air trenches in the arrayed waveguide section.

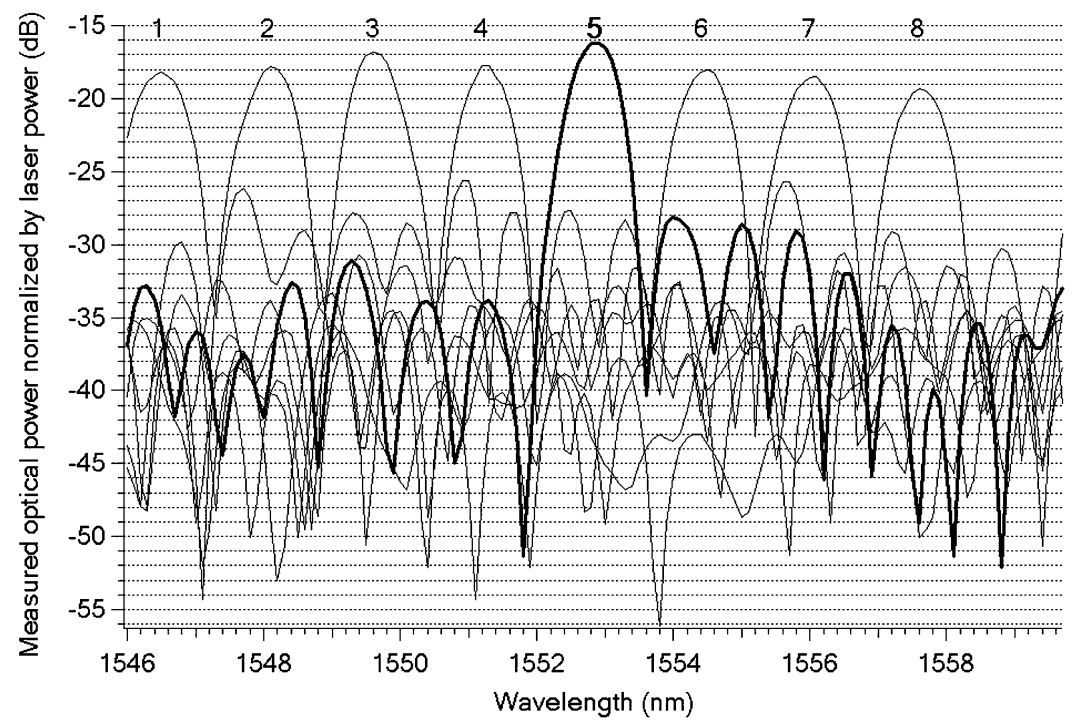

Fig. 10. Typical transmission spectrum of an $8 \times 8$ PFCB air-trench bend AWG. The mainlobes are labeled for each output waveguide. The line style for Output 5 is bold to permit the sidelobe pattern, which is typical for all of the outputs, to be observed. Noted that the main crosstalk tends to arise from adjacent channel sidelobes.

air-trench bend AWG such that the thermooptical effect [which in a single waveguide is proportional to $\Delta n(T) *$ (pathlength)] does not change as much as in a conventional AWG that has much longer path lengths in the arrayed waveguides. Hence, the optical path difference between waveguides in the arrayed waveguide region has less dependence on temperature and there is correspondingly less wavelength shift. As a final comment, note in Fig. 12 that the insertion loss increases when the temperature is raised. This is largely owing to thermally dependent misalignment in the fiber butt coupling.
The temperature-dependence measurement result reveals an important advantage of air-trench bend AWGs in that the device thermal shift is significantly reduced by shrinking the device dimensions. This lowers the environmental requirements for device operation, such as precise temperature control and effectively decreases the device packaging cost, which is the major part of the final device cost. As mentioned before, the results reported in this paper should be obtainable in other low index and low index contrast (LILIC) material systems, such as silica. 


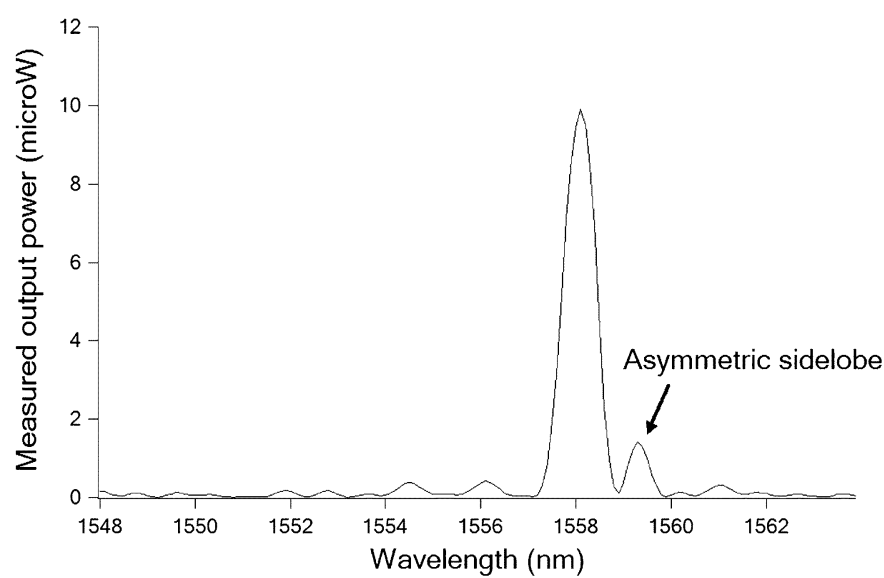

Fig. 11. Typical spectral response for a single AWG output waveguide.

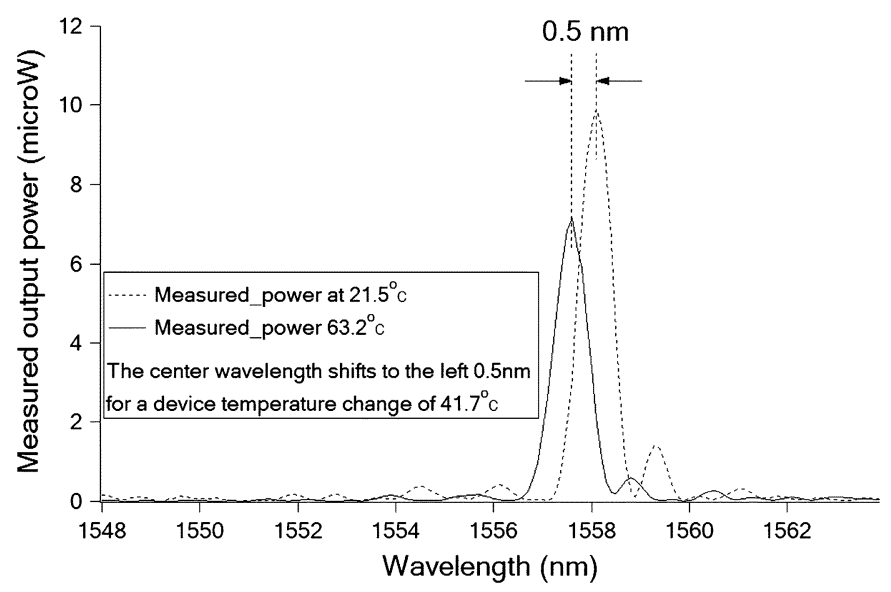

Fig. 12. Measured transmission spectrum of a center channel at room temperature $\left(21.5^{\circ} \mathrm{C}\right)$ and at a higher temperature $\left(63.2{ }^{\circ} \mathrm{C}\right)$.

\section{Polarization Dependence}

As shown in Fig. 13, the measured polarization-dependent wavelength shift is $1.3 \mathrm{~nm}$. This value is related to the PFCB film birefringence $\left(n_{\mathrm{TE}}-n_{\mathrm{TM}}=2.0 \times 10^{-3}\right)$. For a conventional AWG, we can expect a polarization-dependent wavelength shift of $2.1 \mathrm{~nm}$ based on [23]

$$
\Delta \lambda=\frac{\Delta n \Delta L}{m}
$$

where $\Delta n$ is the material birefringence, $m=110$ is the diffraction order, and $\Delta \mathrm{L}=114.923 \mu \mathrm{m}$ is the path difference of the arrayed waveguides. The measured polarization-dependent wavelength shift for the air-trench bend AWG is smaller than that of conventional AWG made with the same materials. This is again partially due to the shorter paths in the arrayed waveguides of the air-trench bend AWG.

\section{CONCLUSION}

An ultracompact AWG for wavelength division multiplexing using air-trench $45^{\circ}$ bends has been demonstrated with PFCB waveguides on a polyimide wafer. Air-trench $45^{\circ}$ bends are used in both the arrayed waveguide region and the input/output

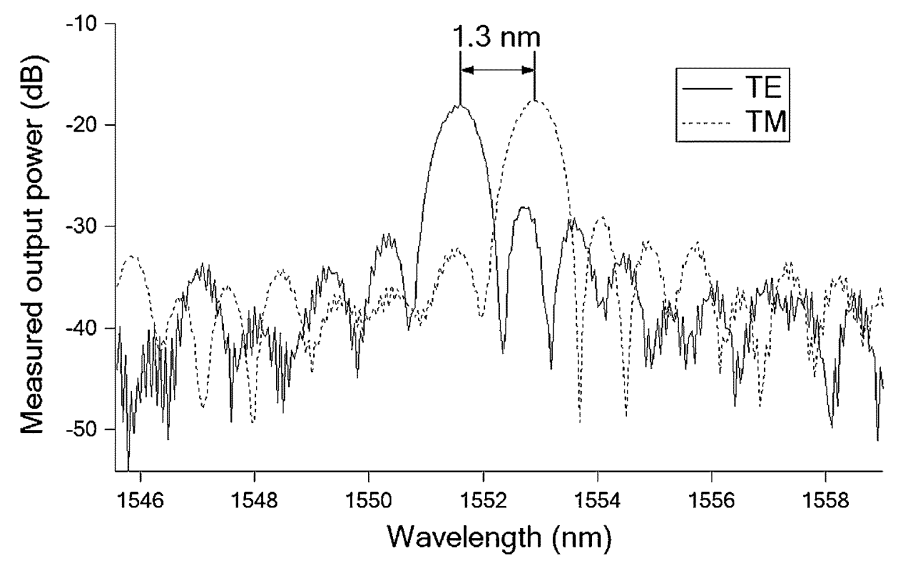

Fig. 13. Measured polarization-dependent wavelength shift.

waveguide region to totally avoid curved waveguide bends to realize the smallest possible AWG device. The chip area for an air-trench bend AWG occupies only 1/20 of the conventional type. The wavelength thermal shift of the AWG device has been significantly reduced by shrinking the device dimensions.

\section{REFERENCES}

[1] M. K. Smit and C. V. Dam, "PHASAR-based WDM-devices: Principles, design and applications," IEEE J. Sel. Topics Quantum Electron., vol. 2, no. 2, pp. 236-250, Jun. 1996.

[2] J. Jiang, C. L. Callender, C. Blanchetiere, J. P. Noad, S. Chen, J. Ballato, and D. W. Smith, Jr., "Property-tailorable PFCB-containing polymers for wavelength division devices," J. Lightw. Technol., vol. 24, no. 8, pp. 3227-3234, Aug. 2006.

[3] T. Mizuno, T. Kitoh, M. Ishii, Y. Inoue, T. Saida, M. Itoh, T. Shibata, and Y. Hibino, "Compact and low-loss arrayed waveguide grating module with tolerance-relaxed spot-size converter," IEEE Photon. Technol. Lett., vol. 15, no. 2, pp. 239-241, Feb. 2003.

[4] T. Suzuki and H. Tsuda, "Ultrasmall arrowhead arrayed-waveguide grating with V-shaped bend waveguides," IEEE Photon. Technol. Lett., vol. 17, no. 4, pp. 810-2, Apr. 2005.

[5] J. Ballato, D. W. Smith, Jr., and S. Foulger, "Optical properties of perfluorocyclobutyl polymers," J. Opt. Soc. Amer. B, Opt. Phys., vol. 20, no. 9, pp. 1838-1843, Sep. 2003.

[6] D. W. Smith, Jr., S. Chen, S. Kumar, J. Ballato, H. Shah, C. Topping, and S. Foulger, "Perfluorocyclobutyl copolymers for microphotonics," Adv. Mater., vol. 14, no. 21, pp. 1585-1589, Nov. 2002.

[7] J. Ballato, S. H. Foulger, and D. W. Smith, Jr., "Optical properties of perfluorocyclobutyl polymers-II: Theoretical and experimental attenuation," J. Opt. Soc. Amer. B, Opt. Phys., vol. 21, no. 5, pp. 958-967, May 2004.

[8] J. Gordon, J. Jin, D. Smith, and J. Ballato, "Optical properties of perfluorocyclobutyl polymers. III. spectroscopic characterization of rare earth doped PFCB," J. Opt. Soc. Amer. B, vol. 22, pp. 1654-1659, 2005.

[9] G. Kumar, C. Chen, R. Riman, S. Chen, D. Smith, and J. Ballato, "Optical properties of a transparent $\mathrm{CaF}_{2}: \mathrm{Er}^{3+}$ Fluoropolymer Nanocomposite," Appl. Phys. Lett., vol. 86, 2005, Article 241105.

[10] G. A. Kumar, R. E. Riman, S. Banerjee, A. Kornienko, J. G. Brennan, S. Chen, D. Smith, and J. Ballato, "Infrared fluorescence and optical gain characteristics of chalcogenide-bound erbium cluster-fluoropolymer nanocomposites," Appl. Phys. Lett., vol. 88, 2006, Article 091902.

[11] G. A. Kumar, C. W. Chen, J. Ballato, and R. E. Riman, "Optical characterization of infrared emitting rare-earth-doped fluoride nanocrystals and their transparent nanocomposites," Chem. Mater., vol. 19, pp. 1523-1528, 2007.

[12] A. Neilson, S. Iacono, S. Budy, J. Ballato, and D. Smith, "Mixed chromophore perfluorocyclobutyl (PFCB) co-polymers for tailored light emission," Macromolecules, 2007, submitted for publication.

[13] G. Chen, R. Rapaport, D. Fuchs, L. Lucas, A. Lovinger, and S. Vilan, "Optical gain from InAs nanocrystal quantum dots in a polymer matrix," Appl. Phys. Lett., vol. 87, no. 25, 2005, Art. No. 251108. 
[14] S. Suresh, S. Chen, J. Ballato, L. M. Hayden, A. Abler, and D. Smith, "Synthesis and characterization of perfluorocyclobutyl (PFCB) polymers containing polyene chromophores," J. Polym. Sci.B, Polym. Phys., 2007, submitted for publication.

[15] J. Jin, D. Smith, S. Glasser, D. Perahia, S. Foulger, J. Ballato, K. Kong, and S. Kumar, "Liquid crystalline perfluorocyclobutyl (PFCB) aryl ether polymers containing oligophenylene mesogens," Macromolecules, vol. 39, pp. 4646-4649, 2006.

[16] L. Li, G. P. Nordin, J. M. English, and J. Jiang, "Small-area bends and beamsplitters for low-index-contrast waveguides," Opt. Express, vol. 11, no. 3, pp. 282-290, 2003.

[17] J. Cardenas, L. Li, S. Kim, and G. P. Nordin, "Compact low loss single air interface bends in polymer waveguides," Opt. Express, vol. 12, no. 22, pp. 5314-5324, 2004.

[18] Y. Lin, J. Cardenas, S. Kim, and G. P. Nordin, "Reduced loss through improved fabrication for single air interface bends in polymer waveguides," Opt. Express, vol. 14, no. 26, pp. 12803-12813, 2006.

[19] N. Rahmanian, S. Kim, and G. P. Nordin, "Anisotropic high aspect ratio etch development for perfluorcyclobutyl polymers with stress relief technique," J. Vac. Soc. Am. B, vol. 24, no. 6, pp. 2572-2577, 2006.

[20] J. Jiang, C. L. Callender, C. Blanchetière, J. P. Noad, S. Chen, J. Ballato, and D. W. Smith, Jr., "Arrayed waveguide gratings based on perfluorocyclobutane polymers for CWDM applications," IEEE Photon. Technol. Lett., vol. 18, no. 2, pp. 370-372, Jan. 2006.

[21] E.-S. Kang, W.-S. Kim, D.-J. Kim, and B.-S. Bae, "Reducing the thermal dependence of silica-based arrayed-waveguide grating using inorganic-organic hybrid materials," IEEE Photon. Technol. Lett., vol. 16, no. 12, pp. 2625-2627, Dec. 2004.

[22] N. Keil, H. H. Yao, C. Zawadzki, J. Bauer, M. Bauer, C. Dreyer, and J. Schneider, "Athermal all-polymer arrayed-waveguide grating multiplexer," IEEE Electron. Lett., vol. 31, no. 9, pp. 579-580, Apr. 26, 2001.

[23] F. Wang, K. Chen, W. Sun, H. Zhang, C. Ma, M. Yi, S. Liu, and D. Zhang, "32-Channel arrayed waveguide grating multiplexer using low loss fluorinated polymer operating around $1550 \mathrm{~nm}$," Opt. Commun., vol. 259, pp. 665-669, Mar. 15, 2006.

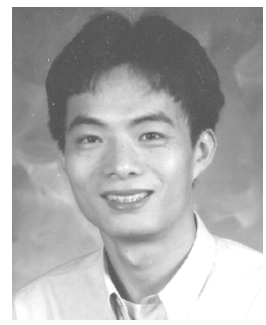

Yongbin Lin received the B.S. degree in chemical engineering and photosensitive chemistry from the East China University of Science and Technology, Shanghai, China, in 1992 and the M.S. degree in applied optics from the Rose-Hulman Institute of Technology, Terre Haute, IN, in 2002, and the Ph.D. degree in optical science and engineering from the University of Alabama in Huntsville in 2007.

$\mathrm{He}$ is a Research Scientist of the Nano and Micro Device Center (NMDC) at the University of Alabama in Huntsville. His research interests to date lie in planar lightwave circuit integration using air-trench bends and splitters, and waveguide chemical and bio sensors based on the refractive index variation detection.

Nazli Rahmanian, photograph and biography not available at the time of publication.
Seunghyun Kim received the B.S. degree in physics from Suwon University, Suwon, South Korea, in 1998 and the Ph.D. degree in optical science and engineering from the University of Alabama in Huntsville in 2004.

$\mathrm{He}$ is currently a Research Associate at Brigham Young University, Provo, UT. His research interests are photonic biological chemical sensors and integrated photonic devices.

Gregory P. Nordin (M'92) received the B.S. degree in physics from Brigham Young University, Provo, UT, in 1984, the M.S. degree in physics from the University of California at Los Angeles (UCLA) in 1986, and the Ph.D. degree in electrical engineering from the University of Southern California, Los Angeles, in 1992.

From 1984 to 1992, he was employed at Hughes Aircraft Company. From 1992 to 2005, he was at the University of Alabama in Huntsville and was the Founding Director of the university's Nano and Micro Devices Center. $\mathrm{He}$ joined Brigham Young University in 2005, where he holds the Engineering and Technology Endowed Chair and is Professor of Electrical and Computer Engineering.

Chris Topping received the Ph.D. degree in chemistry from the University of Hull, U.K., in 1998 for his work with Prof. S. Woodward on the design and synthesis of chiral ligands for catalytic asymmetric synthesis.

He subsequently worked for two years as a Postdoctoral Research Fellow in organic chemistry with Prof. K. Dieter at Clemson University, Clemson, SC, before joining Prof. Smith's polymer research group in order to develop novel semi-fluorinated perfluorocylcobutyl polymers (based on trifluorovinylether monomers) for a variety of optical and other high-tech applications. He is currently at Tetramer Technologies, LLC, Pendleton, SC, where he is responsible for development, scale-up, process development, and production of materials for optical, fuel cell, gas separation, and renewable resource applications. In addition, he also retains the position of Research Assistant Professor at Clemson University.

Dennis W. Smith, Jr., photograph and biography not available at the time of publication.

John Ballato received the B.S. degree in ceramic science and engineering and the $\mathrm{Ph} . \mathrm{D}$. degree in ceramic and materials engineering from Rutgers University, Piscataway, NJ, in 1993 and 1997, respectively.

$\mathrm{He}$ is a Professor of materials science and engineering at Clemson University, Clemson, SC, where he also directs COMSET, the Center for Optical Materials Science and Engineering Technologies, which is a South Carolina Research Center of Economic Excellence. He has published over 120 archival scientific papers, holds 14 U.S. and foreign patents, has given in excess of 110 invited lectures/colloquia, and has co-organized 20 national and international conferences and symposia. 